\title{
Sloshing Analysis of a Heterogeneous Viscous Liquid in Immovable Tank under Pitching Excitation
}

\author{
J. El Bahaoui ${ }^{\dagger}$, H. Essaouini and L. El Bakkali \\ Abdelmalek Essadi University, Faculty of sciences, Department of Physics, Laboratory M2SM, Tetuan, \\ Morocco
}

$\dagger$ Corresponding Author Email: jelbahaoui@yahoo.com

(Received May 26, 2019; accepted January 25, 2020)

\begin{abstract}
The effects of the heterogeneity of liquid on the tank sloshing under pitching excitation are analyzed and discussed. The time history of the free surface elevation for tank containing a homogeneous - heterogeneous liquid are recorded and discussed. Numerical simulations are performed for various functions of density using the finite-element method. A theoretical model in the case of heterogeneous viscous liquid are developed using the variation formulation based on the Navier-Stokes equations. The effect of viscosity on the responses is also discussed for each case. In each case, the time history plots for the vertical fluid displacement at a select node, and the pressure in selected elements are presented to illustrate the results of numerical simulations. The effect of heterogeneity parameter of the amplitude of liquid sloshing in a two dimensional partially filled rectangular tank under pitch excitation is conducted to investigate the effects of excitation variable density on the liquid sloshing by a series of numerical experiments. The results are compared with existing theoretical study and the comparison shows fair agreement.
\end{abstract}

Keywords: Heterogeneous and viscous Liquid; Numerical simulation; Variational method; Sloshing analysis; Pitching excitation.

\section{INTRODUCTION}

The sloshing of fluid can cause a many of instability problems in immovable tanks. The liquid storage tank is widely used in our life and constitutes an important component in the as water distribution system, the vehicle fuel tank and petroleum plants etc... For example in the case of the vehicle fuel tank, the problem is to model the reduce of sloshing in the tanks to avoid the noise. The sloshing caused by the movement of the tank affect stability of the system severely. The sloshing of liquid constitutes a broad class of problems of practical importance with regard to ensure the safety of liquid transportation. Over the years, the problem of sloshing has been studied by a number of researchers by using different methods and experimental technology. (Eswaran and Ujjwal Saha 2011) presents a review of experimental investigation in the sloshing of liquids in partially filled tanks, this work attempts to give a review of some selected experimental investigations carried out during the last couple of decades. Numerical simulation approach of sloshing in 2D rectangular tanks based on the prediction of free surface was studied by Zhang and Sun (2014) and Zhang et al.
(2016), a finite difference method for the 2D nonlinear sloshing waves has been developed. (Faltinsen 1978) presented a nonlinear numerical method of 2D sloshing in tanks, also, he has developed the theoretical approaches to predict the behavior of the free surface elevation. (Chen and Huang 2015) presented in his work the simulation of the complete 2D sloshing motion by a finite difference method. (Goudarzi and Sabbagh-Yazdi 2012) developed the numerical mode using the finite elements method for investigate of nonlinear sloshing effects in seismically excited tanks, the linear theory is briefly introduced, the nonlinear numerical model based on volume of fluid (VOF) method for tracking free surface is presented, also the experimental work was carried for results validation. Recently, (Goudarzi and Nourae Danesh 2016) presents the numerical investigation of vertically baffled rectangular tank under seismic excitation. The numerical models are validated against each other and available analytical solution from Faltinsen (1974) and experimental data from Liu and Lin (2008) (Pal 2010). An experimental and CFD analysis of sloshing in tanks has been developed by (Brar and Singh 2014), this study is focused on the fluid structure interactions in 
elliptical tank. Cho et al. (2005) carried out a numerical method to analyze the resonance characteristics of liquid sloshing in a $2 \mathrm{D}$ baffled tank. They cannot resolve the viscous and the rotational motion of the liquid sloshing because of the potential flow theory. The multiphase CFD modelling has been utilized to investigate the liquid sloshing (Godderidge, 2009), they showed good agreement with the experimental data. (Nakayama and Washizu 1980) adopted the boundary element method to analyze the sloshing in the tank subjected to pitching oscillation. The study of the classical case of the system formed by the rigid body containing the homogeneous ideal liquid, by means of the methods of functional analysis, has been the subject of very many works see, for example (Capodanno 1996), (Kopachevskii 2003). On the other hand, the case of the heterogeneous incompressible liquid in the container was studied, first by Rayleigh and then, was the subject of limited number of works (Capodanno 1993; 2001);( Lamb 1932) and (Essaouini 2014). A twodimensional numerical model is developed by Elahi et al. (2015) to study liquid sloshing in containers in presence of water/air free surface deformation, liquid viscosity and surface tension. The model is validated by a comprehensive comparison between the computational and theoretical/experimental results for various time-dependent linear/angular acceleration sloshing scenarios. A computational model has been developed that can simulate liquid sloshing in 2D containers and fuel tanks with angular and translational movement including linear/angular accelerations. The model can be used to determine forces and torques that are exerted to the structure of vessels or containers due to the sloshing waves. (Javanshir et al. 2013) present the Numerical Simulation of liquid Sloshing with baffles in the fuel container, a two-dimensional numerical model is developed to study viscous liquid sloshing in a tank with internal baffles. The governing equations for the 2D incompressible fluid flow are continuity and Navier-Stokes equations along with an equation for the free surface advection. The deformation of the liquid-gas interface is modelled using the Volume-of-Fluid (VOF) method. (Veldman et al. 2007) study is the influence of sloshing liquid on the dynamics of spacecraft. A combined theoretical and experimental approach has been followed. On the one hand, CFD simulations have been carried out to predict the combined liquid/solid body motion. The modeling of free surface flows in presence of an arbitrary moving object are presented by (Mirzai 2012 ), a numerical algorithm is developed for simulating the interactions between a liquid and a solid object in presence of a free-surface. This model is the fast-fictitious-domain method integrated into the volume-of-fluid (VOF) technique used for tracking the free surface motion.

In this work, our study is about liquid heterogeneity regarding density and viscosity of them, the authors study the effect of the variable density liquid of sloshing in partially filled rectangular tank under pitch excitation, the liquid whose density is generally nonlinear function of the height. To study the effect of the variable density the fluid is assumed homogeneous in the first time and heterogeneous in the second time, isotropic, viscous and Newtonian and exhibits only limited compressibility. A theoretical model and mathematical formulation in the case of heterogeneous viscous liquid are developed using the variation formulation, to solve the equations generated by this model, a 2D modeling was performed with COMSOL-Multiphysics.

\section{MATHEMATICAL FORMULATION IN THE CASE OF HETEROGENEOUS VISCOUS LIQUID}

\subsection{The Case of Immovable Container with Heterogeneous Viscous liquid}

We denote with $\overrightarrow{\mathbf{u}}(x, t)$ the small displacement of a particle of the liquid, which occupies the position $x$ at the instant $t$ from its equilibrium position. $\rho^{*}(x, t), P^{*}(x, t)$ are respectively the density and pressure.

The coefficient of viscosity of the liquid at constant temperature $\mu^{*}$ is function of the density $\rho^{*}$, we have $\mu^{*}=\mu^{*}\left(\rho^{*}\right)$, The linearized Navier-Stokes equation of the motion of the liquid can be written in the following form (Essaouini et al. 2014) and (Lyu et al. 2017):

$\left\{\begin{array}{l}\rho^{*} \ddot{u}_{\mathrm{i}}=-\frac{\partial P^{*}}{\partial x_{\mathrm{j}}}+\frac{\partial}{\partial x_{\mathrm{i}}}\left(2 \mu^{*}\left(\rho^{*}\right) \varepsilon_{\mathrm{ij}}(\dot{\overrightarrow{\mathbf{u}}})\right)-\rho^{*} g \delta_{\mathrm{i} 3} \quad(\mathrm{i}=1,2,3) \\ \operatorname{div}(\dot{\overrightarrow{\mathbf{u}}})=0 \quad \text { in } \Omega \text { (incompressibility) }\end{array}\right.$

Where ${ }_{\varepsilon_{\mathrm{ij}}}(\dot{\overrightarrow{\mathbf{u}}})=\frac{1}{2}\left(\frac{\partial \dot{\mathrm{u}}_{\mathrm{i}}}{\partial x_{\mathrm{j}}}+\frac{\partial \dot{\mathrm{u}}_{\mathrm{j}}}{\partial x_{\mathrm{i}}}\right) \quad$ are the components of the tensor of the velocities of strain, we must add the continuity equation:

$$
\frac{\partial \rho^{*}}{\partial t}+\dot{\vec{u}} \cdot \overrightarrow{\operatorname{grad}} \rho^{*}=0 \quad \text { in } \Omega
$$

and the Incompressibility equation

$$
\operatorname{div}(\overrightarrow{\mathbf{u}})=0 \quad \text { in } \Omega
$$

- Now we consider the boundary conditions:

i) The no-slip condition at the rigid wall $S$ is:

$\overrightarrow{\mathbf{u}}_{\mid S}=0$

ii) The dynamic conditions on the moving free surface $\Gamma_{t}$ takes the form

$\sum_{i j}\left(\dot{\overrightarrow{\mathbf{u}}}, P^{*}\right) \mathrm{n}_{\mathrm{t}_{\mathrm{j}}}=-p_{a} \mathrm{n}_{\mathrm{t}_{\mathrm{j}}} ;$ on $\Gamma_{t}$ 
Where $p_{a}$ is the atmospheric pressure, which is assumed constant. In linear theory, this equation can be written in the present form

$$
-\left(P_{\Gamma_{t}}^{*}-p_{a}\right) \mathrm{n}_{\mathrm{i}}+2 \mu^{*} \varepsilon_{\mathrm{ij}}(\dot{\overrightarrow{\mathbf{u}}}) \mathrm{n}_{\mathrm{j}}=0 \text {, on } \Gamma
$$

We have finally

$$
\left\{\begin{array}{l}
\varepsilon_{13}(\dot{\overrightarrow{\mathbf{u}}})=0, \varepsilon_{23}(\dot{\overrightarrow{\mathbf{u}}})=0 \\
-\left(P_{\Gamma_{t}}^{*}-p_{a}\right)+2 \mu^{*}\left(\rho^{*}\right) \varepsilon_{33}(\dot{\overrightarrow{\mathbf{u}}})=0
\end{array}\right.
$$

In the equilibrium position we have :

If $\rho_{0}$ and $P_{s t}$ are the density and the pressure of the liquid in the equilibrium position, we have

$\overrightarrow{\operatorname{grad}} P_{s t}=-\rho_{0} g \vec{x}_{3}$

so that $P_{s t}$ and $\rho_{0}$ are functions of $x_{3}$, with

$\frac{\mathrm{d} P_{s t}\left(x_{3}\right)}{\mathrm{d} x_{3}}=-\rho_{0}\left(x_{3}\right) g$,

We have

$$
P_{s t}=-g \int_{0}^{x_{3}} \rho_{0}(w) \mathrm{d} w+p_{a}
$$

Setting

$$
R_{0}\left(x_{3}\right)=\int_{0}^{x_{3}} \rho_{0}(w) \mathrm{d} w,
$$

We can write

$$
P_{s t}=-g R_{0}\left(x_{3}\right)+p_{a},
$$$$
\text { and } \quad R_{0}(0)=0, R_{0}^{\prime}\left(x_{3}\right)=-\rho_{0}\left(x_{3}\right)
$$

Finally the Navier - Stokes equations take the form:

$\rho^{*} \ddot{\mathrm{u}}_{\mathrm{i}}=\frac{\partial \sigma_{\mathrm{ij}}}{\partial x_{\mathrm{j}}}-\left(\rho^{*}-\rho_{0}\left(x_{3}\right)\right) g \delta_{\mathrm{i} 3} \quad$ in $\Omega$

where $p(x, t)=P^{*}(x, t)-P_{s t}$, is the dynamic pressure, and $\sigma_{\mathrm{ij}}=-p \delta_{\mathrm{ij}}+2 \mu^{*} \varepsilon_{\mathrm{ij}}(\dot{\overrightarrow{\mathbf{u}}})$.

The variational formulation of this problem is
$W=\left\{\overrightarrow{\mathbf{v}} / \operatorname{div}(\overrightarrow{\mathbf{v}})=0, \overrightarrow{\mathbf{v}}_{\mid S}=0\right\}$

\subsection{The Case of the Container under Pitching Excitation}

In this paragraph we suppose that the tank has been forced in pitching motion around the midpoint at the at the bottom of the tank, where $\theta, \omega$ represent roll angle, and roll velocity. A and $f$ denote the amplitude

and frequently of the excitation, respectively. Suppose the tank undergoes the external excitation:

$$
\begin{aligned}
& \boldsymbol{\theta}=\boldsymbol{A} \sin (2 \pi \boldsymbol{f t}) \\
& \boldsymbol{\omega}=2 \boldsymbol{\pi} \boldsymbol{f A} \cos (2 \pi \boldsymbol{f t})
\end{aligned}
$$

In Fig. 2, XOY is the inertial reference frame and $\boldsymbol{x} \boldsymbol{o y}$ is the reference frame fixed to the tank. $\boldsymbol{u}$, $\boldsymbol{v}$ and $\boldsymbol{w}$ are the velocity components. The relation between absolute velocity $\overrightarrow{\boldsymbol{v}}_{\boldsymbol{a}}$ and relative velocity $\vec{v}_{r}$ is $\vec{v}_{a}=\vec{v}_{r}+\vec{v}_{e}$

Where $\overrightarrow{\boldsymbol{v}}_{\boldsymbol{e}}$ is carrier velocity, and it is expressed as

$\vec{v}_{e}=\vec{v}_{0}+\vec{\omega} \times \vec{r}$

Where $\overrightarrow{\boldsymbol{r}}$ is the radius vector from the origin $\boldsymbol{O}$ to the arbitrary point in fluid domain, $\vec{v}_{\mathbf{0}}$ represents the translation velocity of the point in moving reference frame and $\vec{\omega}$ represents the relative angular velocity.

The relation between absolute acceleration $\overrightarrow{\boldsymbol{a}}_{\boldsymbol{a}}$ and relative acceleration $\overrightarrow{\boldsymbol{a}}_{\boldsymbol{r}}$ is

$\vec{a}_{a}=\vec{a}_{r}+\vec{a}_{e}+\overrightarrow{\boldsymbol{a}}_{c}$

Where $\overrightarrow{\boldsymbol{a}}_{\boldsymbol{e}}$ and $\overrightarrow{\boldsymbol{a}}_{\boldsymbol{c}}$ represent the carrier acceleration and Coriolis acceleration respectively, and they can be written as

$\vec{a}_{e}=\frac{d \vec{v}_{0}}{d t}+\frac{d \vec{\omega}}{d t} \times \vec{r}+\vec{\omega} \times(\vec{\omega} \times \vec{r})$

$\overrightarrow{\boldsymbol{a}}_{\boldsymbol{c}}=\mathbf{2}\left(\overrightarrow{\boldsymbol{\omega}} \times \overrightarrow{\boldsymbol{v}}_{\boldsymbol{r}}\right)$

The Navier-Stokes Eq. (1) becomes:

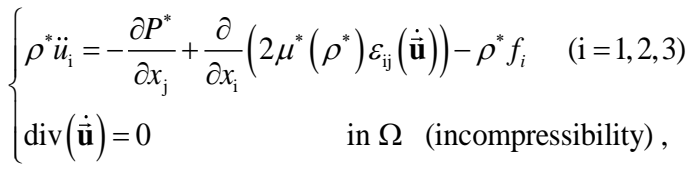

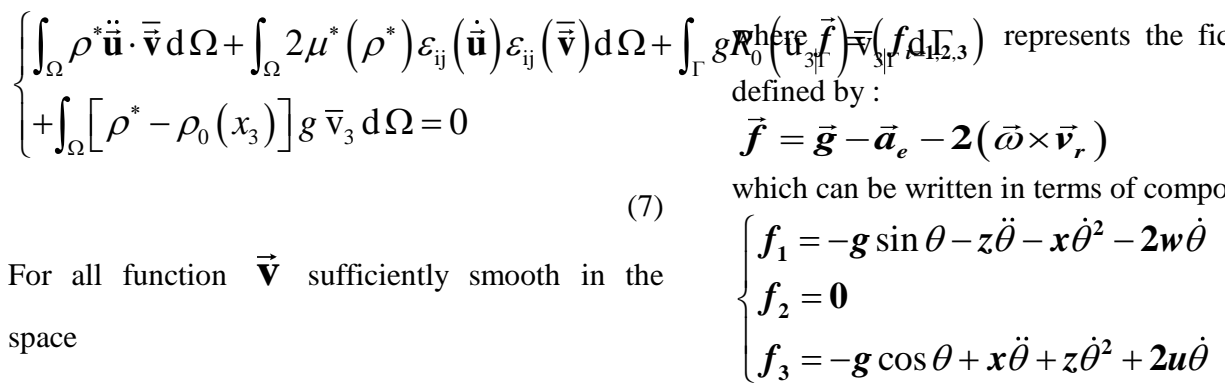




\section{CFD AND NUMERICAL MODEL}

In this paper, the numerical simulations of sloshing have been carried out by using the computational fluid dynamic (CFD) nonlinear program COMSOLMultiphysics that is the good coupled and multiphysics solver. This program is based on the finite element method (FEM), motions are assumed two-dimensional. This 2D model demonstrates the ability of COMSOL-Multiphysics to simulate dynamic free surface to analyze the effect of sloshing modes in the event considered the heterogeneous fluids. In this sense, we can integrate the non-linear shape of the density function. The domain considered here is a rectangular container partially filled with liquid, as shown in Fig. 1.

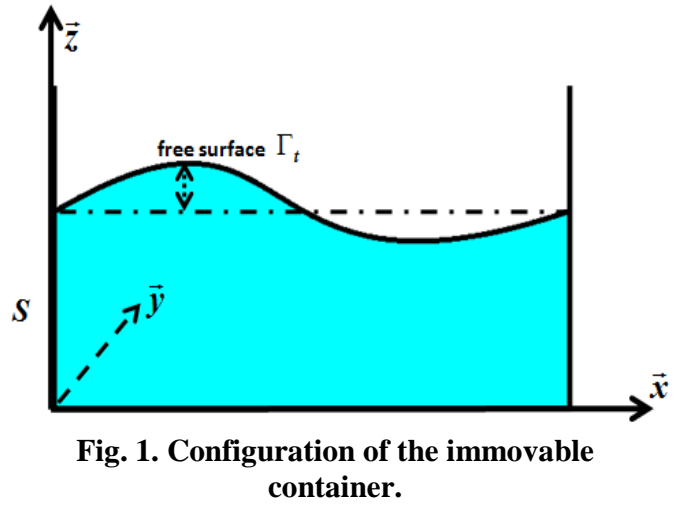

The gravity vector swinging back and forth drives the motion and he surface of the fluid is free to move. The liquid is considered to be incompressible Newtonian, the continuity equation and the momentum equations are given by Eq. (13). This expression may be equated to the local force acting per unit mass of fluid to give the equation of motion in the moving frame. For this consideration, the Eq. (15) is written as follows in the $\mathrm{x}$ and $\mathrm{y}$ directions (Hakan Akyildiz 2012):

$$
\begin{aligned}
& f_{1}=-g \sin \theta-\ddot{\theta} y+\dot{\theta}^{2} x-d\left(\ddot{\theta} \sin \gamma-\dot{\theta}^{2} \cos \gamma\right)-\dot{U} x-\dot{\theta} v \\
& f_{3}=g \cos \theta+\ddot{\theta} x+\dot{\theta}^{2} y-d\left(\ddot{\theta} \cos \gamma+\dot{\theta}^{2} \sin \gamma\right)-\dot{U} y+\dot{\theta} \dot{u}
\end{aligned}
$$

With, $g$ Is the gravitational acceleration, $d$ is the distance between the origin of the moving coordinate and the axes of rotation, $\stackrel{\times}{U} x$ and $\dot{U}_{y}$ are

the acceleration of the tank, the term $2 \dot{\theta} u$ represents the deflecting or carioles force, Finally $u$ and $v$ are the velocity component in the coordinate directions $x$ and $\mathrm{y}$.

In our numerical model, we restrict ourselves in the case only the sinusoidal terms. Then:

$$
\begin{aligned}
& f_{1}=-g \sin \theta \\
& f_{3}=g \cos \theta
\end{aligned}
$$

The tank motion is the pitch oscillations about $\mathrm{x}$ and $\mathrm{y}$-axis only which follows the sinusoidal function given as $\theta(t)=\theta_{o} \sin (\omega t)$ where $\theta_{o}$ and $\omega$ represent the rolling amplitude and the frequency, respectively.

The kinematics and dynamic conditions should be satisfied on the free surface. So

$$
\begin{aligned}
& \frac{\partial \eta}{\partial t}+U . \nabla(\eta-z)=0 \\
& P=P_{\text {atm }}
\end{aligned}
$$

Where $\eta$ represents the free surface profile and $\mathrm{P}_{\mathrm{atm}}$ is the air pressure or ullage pressure inside the tank. The surface tension is ignored in this study. Therefore, a no-shear is needed on the free surface.

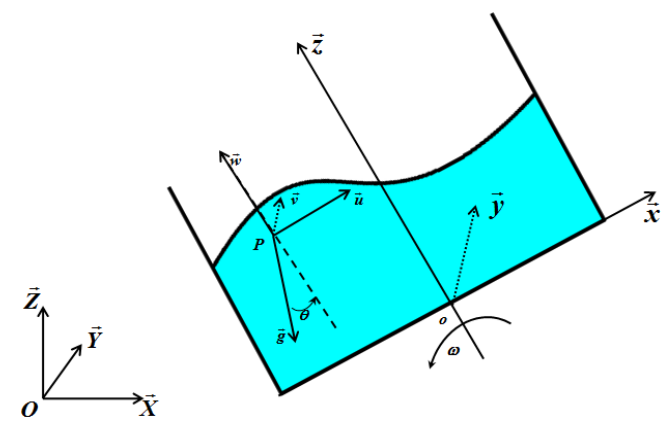

Fig. 2. Configuration of a container under pitching excitation.

\section{MODEL VALIDATION FOR 2-D LIQUID SLOSHING}

\subsection{Liquid Sloshing in the Rectangular Tank under Surge Excitation}

In this section, we shall present the validation of the model presented, the numerical results will be compared to analytical solution and other results. For the cases using COMSOL-Multiphysics, the numerical solution will be compared to other numerical results. The demonstrations of the linear and non-linear 2-D sloshing cases are both present in this part. The linear theory is generally used to evaluate the sloshing free surface elevation in the rectangular tank filled with liquid under the surge excitation. Consider the rectangular tank Fig.2 subjected to surge excitation. For a two dimensional fluid motion and under assumption of excitation amplitude and small fluid response, the tank containing inviscid and irrotational liquid subjected to the periodic displacement $X(t)=D \sin (\omega t), D$ is excitation amplitude, $t$ is time, and $\omega$ is angular frequency of the forced motion.

The equations governing the liquid motion can be expressed in terms

$$
\nabla^{2} \phi=0
$$


$\phi$ is the velocity potential function. The velocity components normal to the fixed boundaries are

$$
\begin{array}{ll}
\frac{\partial \phi}{\partial x}=0 & x= \pm \frac{L}{2} \\
\frac{\partial \phi}{\partial z}=0 & x=-h
\end{array}
$$

$L$ represent the length of the tank and $h$ is the still water depth. The dynamic free surface boundary condition on $z=\eta$ is given by the relation

$$
\frac{\partial \eta}{\partial t}=\frac{\partial \phi}{\partial y} \frac{\partial \eta}{\partial t}-g \eta-\frac{1}{2}[\nabla \phi \times \nabla \phi]-x \ddot{X}(t)
$$

The kinematic free surface boundary condition is expressed by :

$$
\frac{\partial \eta}{\partial t}=\frac{\partial \phi}{\partial y}-\frac{\partial \phi}{\partial x} \frac{\partial \eta}{\partial x}
$$

The total potential function is the sum of perturbed liquid function with the solution of Laplace's problem with nonlinear boundary conditions imposed on the free surface, plus the tank potential function $\phi_{0}=D \omega \cos (\omega t)$ However, the process of evaluating the velocity potential function with the result (Faltinsen 1978 and Goudarzi and SabbaghYazdi 2012)

$$
\begin{aligned}
& \phi=D \omega x \cos \omega t+ \\
& D \sum_{n=0}^{\infty}\left(C_{n} \cos \omega t-\left(C_{n}+\frac{H_{n}}{\omega^{2}}\right) \cos \omega_{n} t\right) \frac{\cosh k_{n}(y+h)}{\cosh k_{n} h} \sin k_{n} x \\
& k_{n}=\frac{(2 n+1)}{L} \pi \\
& \omega_{n}=\sqrt{\left(g k_{n} \tanh k_{n} h\right)} \\
& H_{n}=\omega^{3}\left(\frac{4}{L}\right)\left(\frac{(-1)^{n}}{k_{n}^{2}}\right) \\
& C_{n}=\frac{H_{n}}{\omega_{n}^{2}-\omega^{2}}
\end{aligned}
$$

Where $\omega_{n}$ is the square of the fluid-free-surface natural frequency. The free surface wave height is determined by substituting Eq. (22) into Eq (21), $\eta=\eta_{1}+\eta_{2}$

$$
\begin{aligned}
& \eta=\frac{1}{g} \sum_{n=0}^{\infty} \sin k_{n} x \cosh k_{n} h\left[\begin{array}{l}
-\left(-\frac{\omega}{\omega_{n}^{2}-\omega^{2}}\left(\frac{4 \omega D(-1)^{n}}{L \cosh \left(k_{n} h\right)} \frac{1}{k_{n}^{2}}-\frac{1}{\omega} \frac{4 \omega D(-1)^{n}}{L \cosh \left(k_{n} h\right)} \frac{1}{k_{n}^{2}}\right)\right. \\
\times \omega_{n} \sin \omega_{n} t-\frac{\omega^{2}}{\omega_{n}^{2}-\omega^{2}} \frac{4 D(-1)^{n}}{L \cosh \left(k_{n} h\right)} \frac{1}{k_{n}^{2}} \sin \omega t
\end{array}\right] \\
& -\frac{1}{g} D \omega x \sin \omega t
\end{aligned}
$$

(Wu et al. 1998) have explained the physical behavior of the time-dependent free-surface elevation by considering Eq. (22) which contains two terms, each referring to a wave train. The first component, $\eta_{1}$ corresponds to the excitation frequency $\omega$; the second component, $\eta_{2}$, corresponds to the natural frequencies $\omega_{n}$, and is dominated by $\omega_{0}$. Higher order components in $\eta_{2}$ correspond to the higher natural frequencies, and rapidly decay with increasing $\mathrm{n}$. Hence, $\eta$ is primarily given by the linear sum of waves with frequencies $\omega$ and $\omega_{0}$ as:

The parameter $L$ and $h$ is taken as $0.57 \mathrm{~m}$ and 0.15 $m$ substantially the same dimensions used by, the excitation follows the allowing excitation velocity function: $u=-D \cos (\omega t)$, where $D=a \omega$ is the velocity amplitude with $\mathrm{a}=0.005 \mathrm{~m}$ being the displacement amplitude and the angular frequency $\omega$ is external excitation frequency.

In this section, the numerical results are compared with the analytical solution and the results presented in (Xue and Lin 2011). The time series of free surface elevation of sloshing under surge excitation in deep water are presented in Fig.3. This figure shows the time history of free surface elevation for different excitation frequencies sloshing in the tank without baffle. This phenomena present the liquid sloshing response in the case of the homogeneous liquid in a rectangular tank under surge excitation. The obtained results of the liquid sloshing in the rectangular tank under surge excitation are compared with the results of Xue and Lin (2011) and the numerical results with different excitation frequencies are presented and compared with the linear analytical solution developed by Faltinsen (1978). The comparison between the analytical solution and the numerical results shows that they are in good agreement at the two positions for different excitation frequency. Liu and Lin (2008) showed that the numerical results match well with the experimental measurements. In addition, the linear analytical solution always shows symmetric wave pat-tern; whereas the numerical results can present a typical non-linear wave sloshing phenomenon. Good agreements are obtained in the comparison.

\subsection{Liquid Sloshing in the Rectangular Tank under Pitching Excitation}

In this section, the studies of free surface elevation and amplitude responses of liquid sloshing in filled rectangular tank under pitching excitation are carried using the COMSOL-Multiphysics code. The dimensions of the container are $\mathrm{L}=0.9 \mathrm{~m}$ and $\mathrm{h}=$ $0.6 \mathrm{~m}$.

The container is assumed to be subjected to the following forced sinusoidal pitching oscillation $\theta(t)=\theta_{o} \sin (\omega t)$ about the origin $\mathrm{o}$ in Fig.2. The analytical solution in the case of rectangular tank 

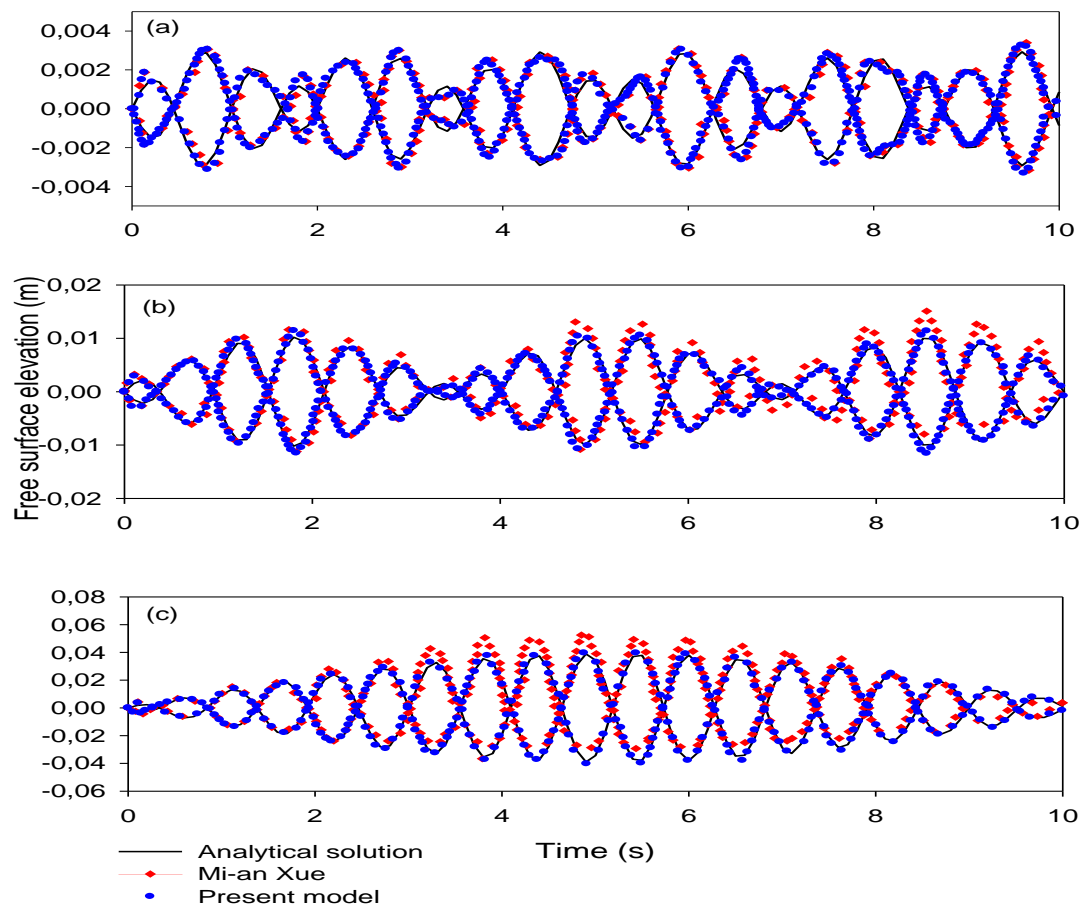

Fig. 3. Comparison of the time history of free surface elevation at $x=+-0.265 \mathrm{~m}$ between the present numerical simulation results, Mi-an Xue (2011) results and linear analytical simulation for different excitation

frequencies: (a) $\omega=0.4 \omega_{0}$ (b) $\omega=0.7 \omega_{0}$, and (c) $\omega=0.9 \omega_{0}$.

under pitching excitation is developed in (Raouf 2005), he expresses the free surface wave height. The Fig. 4 shows the time histories of free surface elevation of the liquid in container, which is forced to pitch about the origin o as given by Eq. (17). The parameters $\theta$ and $\omega$ are assumed so that $\theta_{\mathrm{o}}=0.8 \mathrm{deg}$ and $\omega=5.5 \mathrm{rad} / \mathrm{sec}$, respectively.

The results we have obtained are consistent with the works of Xue and Nakayama. Also, the comparison between the analytical solution and the numerical results shows that they are in good agreement.

\section{RESULTS AND DISCUSSIONS}

We propose in this paragraph to study the physical behavior of a heterogeneous fluid contained in a partially filled rectangular tank. A numerical study using the finite

elements method is presented to evaluate the effect of density on the spectrum sloshing. We present the obtained results in the case of a homogeneous fluid, and that for eventual comparison with the case of a heterogeneous fluid. Three density forms are considered, Case of the Rayleigh density, The Case of the almost homogeneous liquid and sinusoidal density form. The study of fluid structure in two dimensional rectangular tanks is generated in the COMSOL-Multiphysics. The dimensions of tank capacity is fixed in $\mathrm{L}=0.9 \mathrm{~m}, \mathrm{~h}=0.6 \mathrm{~m}$ has been taken into account.
Table 1 Various parameters of the problem

\begin{tabular}{|c|c|c|}
\hline Parameters & Value & Units \\
\hline Dynamics viscosity & Variable & Pa.s \\
\hline Density of liquid & Variable & $\mathrm{Kg} / \mathrm{m}^{3}$ \\
\hline Gravity & 9.814 & $\mathrm{M} / \mathrm{s}^{2}$ \\
\hline $\begin{array}{c}\text { Time taken by the truck to } \\
\text { stop analysis }\end{array}$ & $\begin{array}{c}\text { From } 6 \text { to } \\
20\end{array}$ & $\mathrm{Seconds}$ \\
\hline Excitation frequency & variable & $\mathrm{s}^{-2}$ \\
\hline
\end{tabular}

To study the phenomenon using FEM, the triangular elements for mesh is considered. The table 1 shows the various parameters, these parameters are referred from the literature and present the real situations. The numerical analysis was conducted without any baffles and using the homogeneous fluid in the first time, in the second time the effect of heterogeneity will be considered.

\subsection{Liquid Sloshing Analysis in the Tank under Pitching Excitation}

\section{a) the Case of the Homogeneous Liquid}

In this section, the modeling was conducted to study the physical behavior of a homogeneous liquid and finding the shape of the free surface depending on the viscosity.

The triangular tank subjected to the pitch excitation $\theta(t)=\theta_{0} \sin (\omega t)$ is taken as a numerical example, 
where $\mathrm{L}=0.9 \mathrm{~m}, \mathrm{~h}=0.6 \rho=850 \mathrm{Kg} / 3$, and $\theta_{0}=4^{\circ}$.

The effect of variation of viscosity on the free surface behavior is shown in the Fig,5. The free surface elevation changes as a function of the viscosity augmentation. We can see easily that in the case of highly viscous liquid, the free surface elevation decreased. The mechanical behavior of our liquid can reach the equilibrium position after 30 seconds.

For low viscosity, the slosh motion obviously contains both the excitation and natural frequencies of sloshing in the container, as seen in the top two plots. In the third plot, the viscosity chosen being very high, the sloshing very quickly reaches steady state;

\section{b) The Case of the Rayleigh Density}

Looking at the literature, the majority of work investigation is interested in studying the sloshing problem in the case of homogeneous liquids, but against, in our knowledge, analysis of the effect of heterogeneity is rarely treated. Rayleigh studied the problem of the heterogeneous ideal heavy liquid at first by considering the density of the liquid in equilibrium under the variable nonlinear function at form.

$$
\rho(z)=\rho_{0} \exp (-\beta z)
$$

With $\beta$ is the damping coefficient.

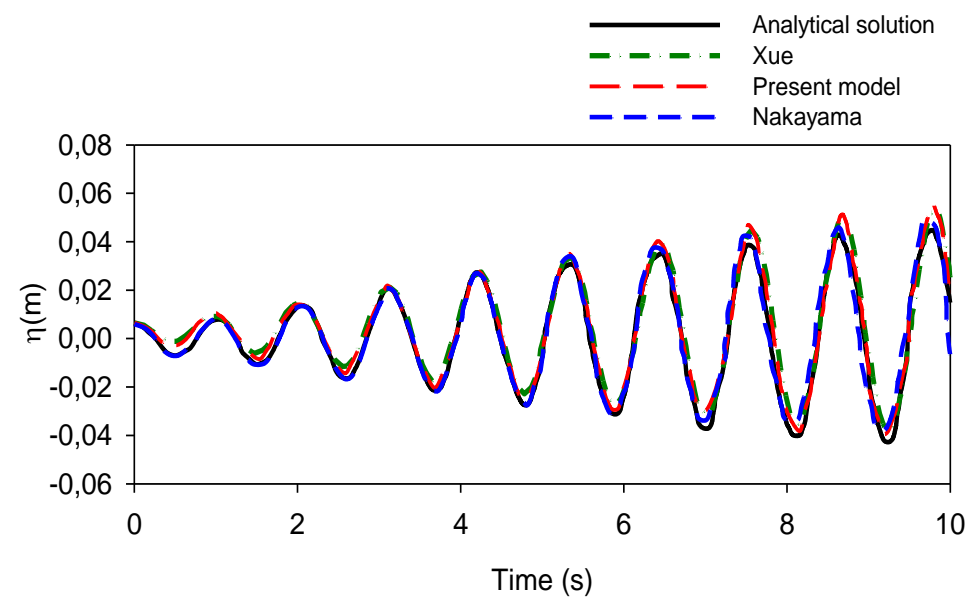

Fig. 4. Comparison of the time histories of free surface elevation at the right wall of the tank for liquid sloshing under pitch excitation among: Nakayama solution, Xue solution, theoretical solution and present numerical result model.

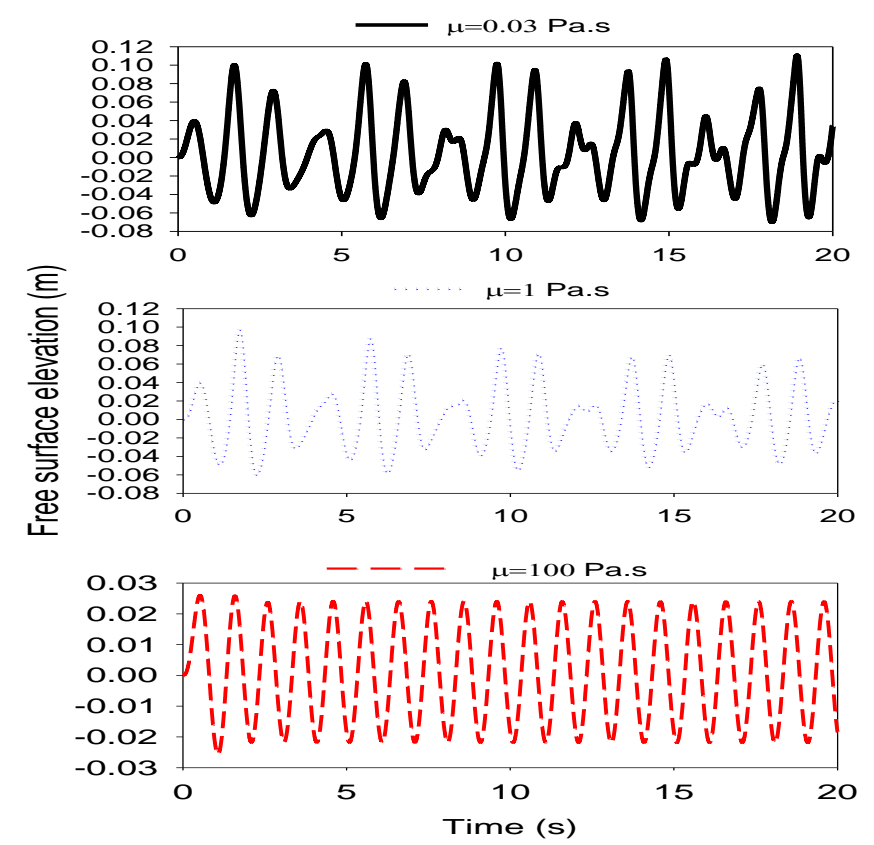

Fig. 5. Time history of relative sloshing wave height for three different coefficients of viscosity ( $\rho=850$ $\mathrm{Kg} / \mathrm{m}^{3}$ ) and $\mathbf{h} / \mathbf{b}$. 

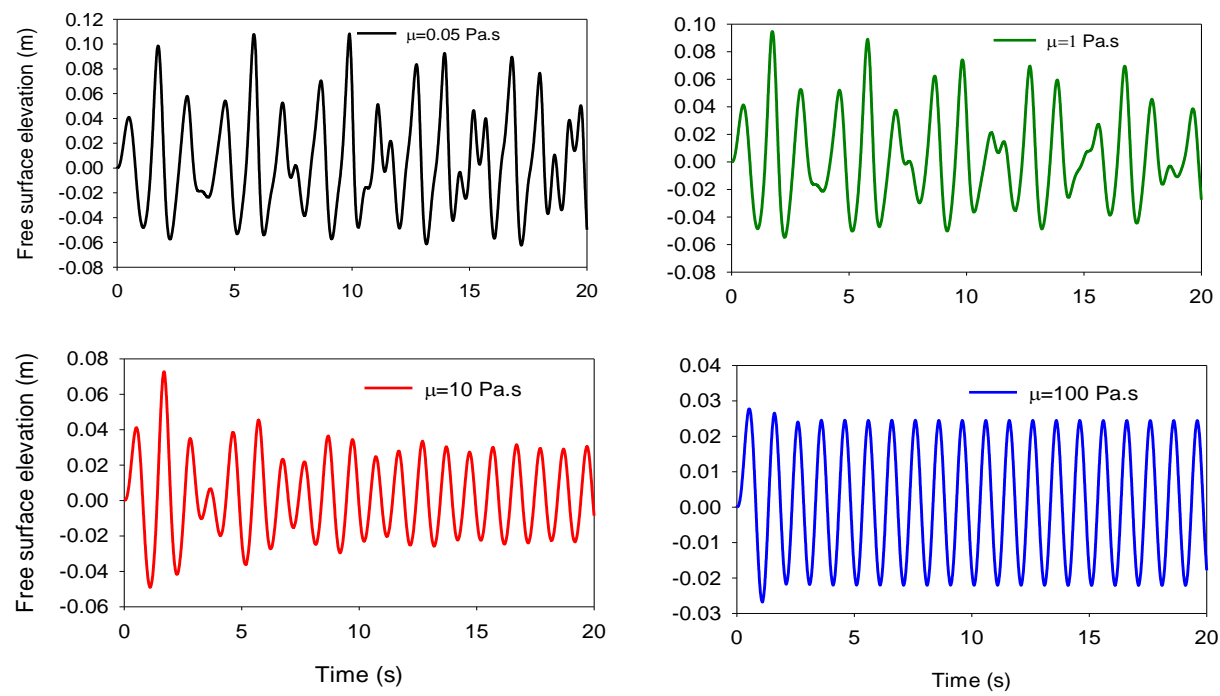

Fig. 6. Time history of relative sloshing wave height for three different coefficients of viscosity $\rho=850$ $\mathrm{Kg} / \mathrm{m}^{3} \beta=0.5, \theta_{0}=4^{\circ}$ and $\mathrm{h} / \mathrm{b}=0.3$.

Figure 6 reveals the effect of Rayleigh density on the free surface elevation in the case of the rectangular tank . The length of this tank is $\mathrm{L}=0.9$ $\mathrm{m}$ and the water depth is $\mathrm{h}=0.6 \mathrm{~m}$. The first result concluded from the Fig. 6, that the free surface elevation is a function of liquid viscosity. If the liquid has a high viscosity, the vibration free surface becomes small. The effect of the Rayleigh density on the free surface was studied, the fig. 6 on the other hand claimed to show sloshing wave heights for three different coefficients of viscosity rather than any mention of Rayleigh density effect on the free surface. The Rayleigh density affected the free surface elevation.

c) The Case of the Almost Homogeneous Liquid

In this section we propose to study the case of the almost homogeneous liquid. The effect on the behavior of the free surface for this heterogeneity will be analyzed; the modeling is to consider the density of fluid as a linear function and may vary depending on the $h$ axis of tank. We suppose that density in equilibrium position in the form: $\rho(z)=\rho_{0}(1-\beta z)$

Let $\mathrm{h}$ be the maximum height of the liquid in the equilibrium position. Where $\rho_{0}$ is positive constants, $\beta$ being sufficiently small so that are negligible with respect to $\beta$ xh. Taking the same conditions as in the case of the homogeneous liquid .The results of the simulation are presented in the Fig.7.

\section{d) The Case of a Sinusoidal Density Form}

We propose in this section a new form of sinusoidal density, the liquid in the tank is considered heterogeneous and its density is of the form:

$$
\rho(z)=\rho_{0} \cos (\beta z)
$$

The parameter $\mathrm{L}$ and $\mathrm{h}$ is taken as $1 \mathrm{~m}$ and $0.6 \mathrm{~m}$, the excitation follows the function $\theta(t)=\theta_{0} \sin (\omega t)$.

The Figure 8 shows the results found. We always conclused that the vibration of free surface elevation is a function of liquid viscosity.

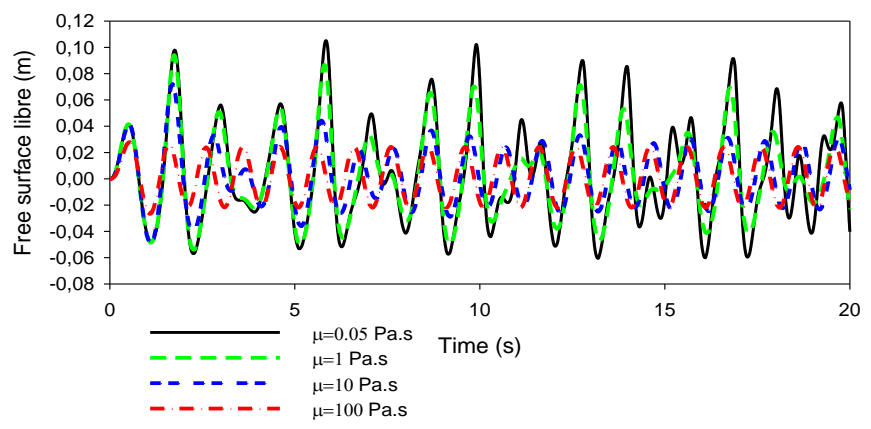

Fig. 7. Numerical results of free surface elevation and the corresponding different viscosity under the pitching excitation $\beta=0.5$ and $\rho_{0}=1000 \mathrm{Kg} / \mathrm{m}$. 

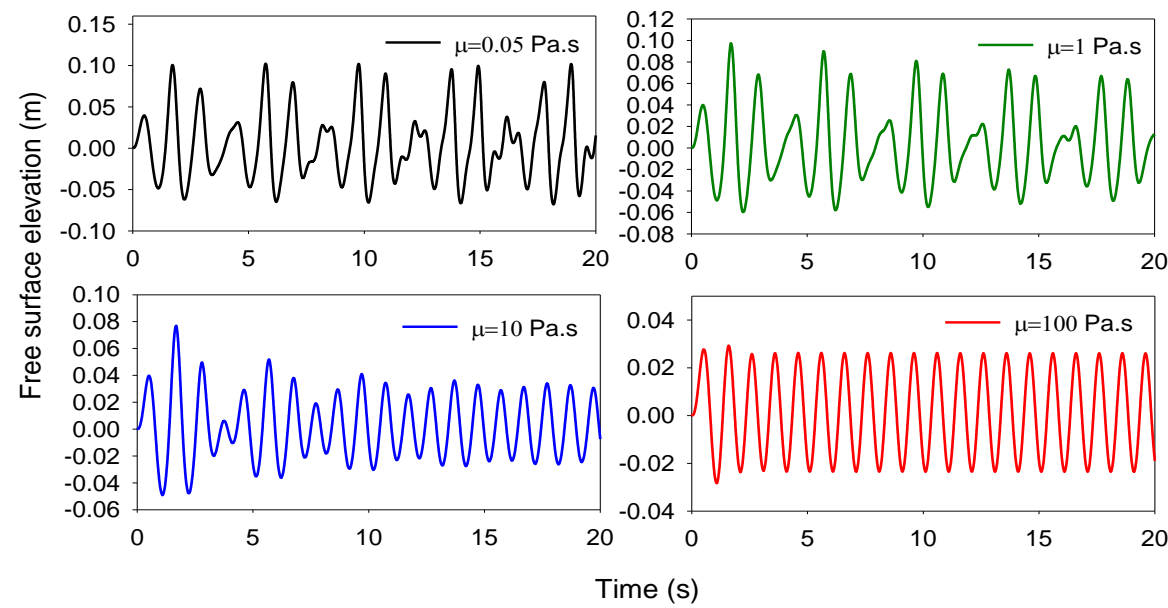

Fig. 8. Numerical results of free surface elevation and the corresponding different viscosity under the pitching excitation $\beta=0.5$ and $\rho_{0}=1000 \mathrm{Kg} / \mathrm{m}^{3}$.

\subsection{Pressure Response to Pitch Excitation with Different Density Form}

In this section, the influence of the density form with various viscosity values on damping effects and characteristics of liquid sloshing in the rectangular tank are numerically investigated. For modeling, we took the same conditions as in paragraph 5.1. The time simulation results of the pressure due to the liquid sloshing at different case of the density are presented.

The Figs. 9, 10, 11 and 12 show the changing pressure results for the different viscosity value for different cases of density. The results show that the density effect is important; the comparative study will be discussed in the following paragraph. A significant pressure increase is observed in the case of liquids with elevated viscosity. The pressure exerted has an influence on the liquid behavior, In the case of the sinusoidal density form, the pressure value is approximately $5 \mathrm{~Pa}$, this value changed to $10 \mathrm{~Pa}$ in the case of a homogeneous liquid. The liquid behavior becomes stable for the high values of the viscosity $\mu=100$ Pa.s. This result is true whatever the density form studied.

5.2 Effect of the Heterogeneity of the Liquid on the Behavior of the Free Surface Profiles

In this section we propose to present the comparative study to show the effect of heterogeneity of the liquid on the behavior of the free surface profiles. The density of our liquid is assumed necessarily variable and depends on the physical properties of the fluid .We changed the beta parameter to see its effect on the variation of the free surface elevation. The results are shown in Figs. 13, 14 and 15.
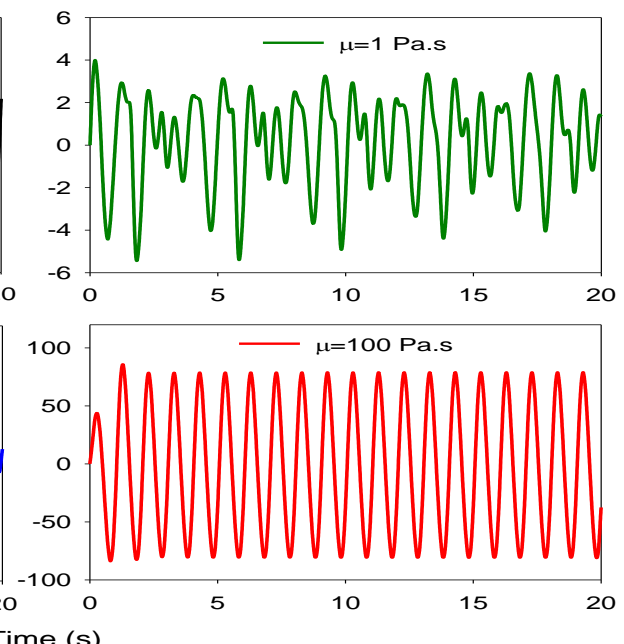

Fig. 9. Changing pressure results for the different viscosity value (Case of homogeneous liquid). 


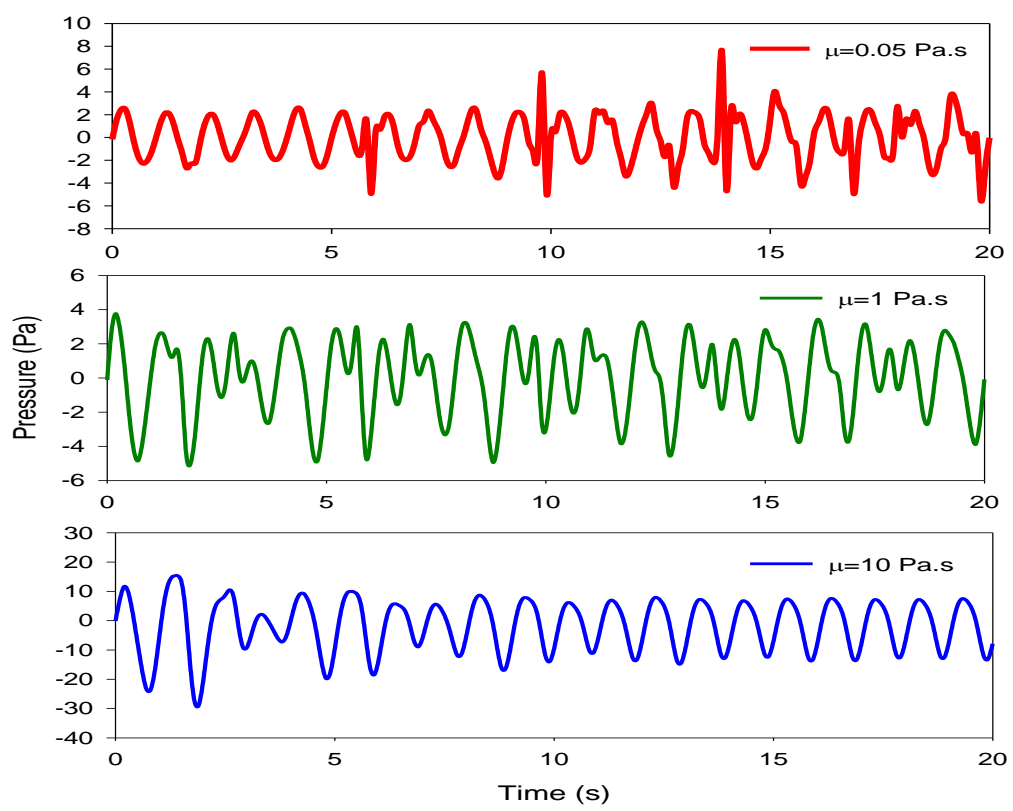

Fig. 10. Changing pressure results for the different viscosity value (The case of the Rayleigh density).

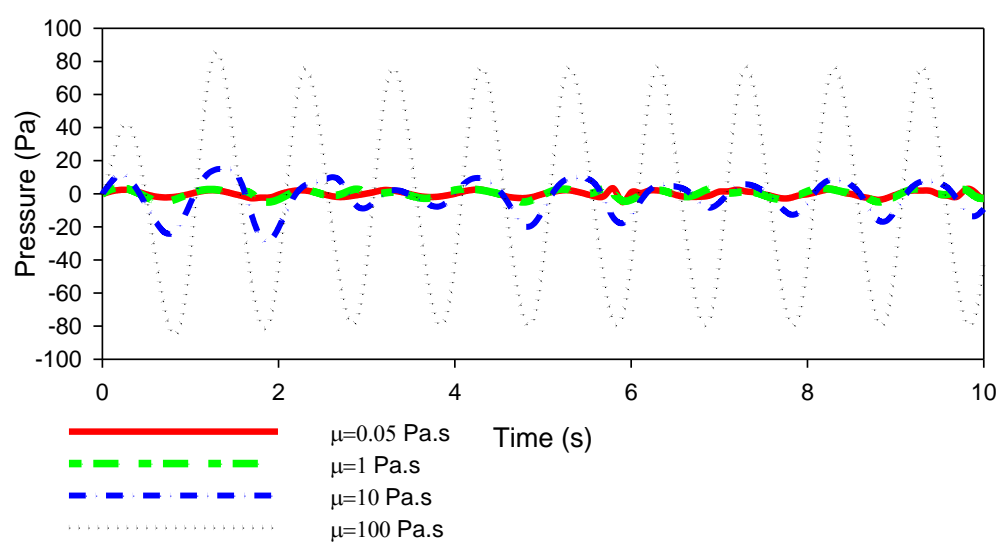

Fig. 11. Changing pressure values for the pitch angle $\theta_{0}=4^{\circ}$ at the different values of viscosity. The Case of the almost homogeneous Liquid.

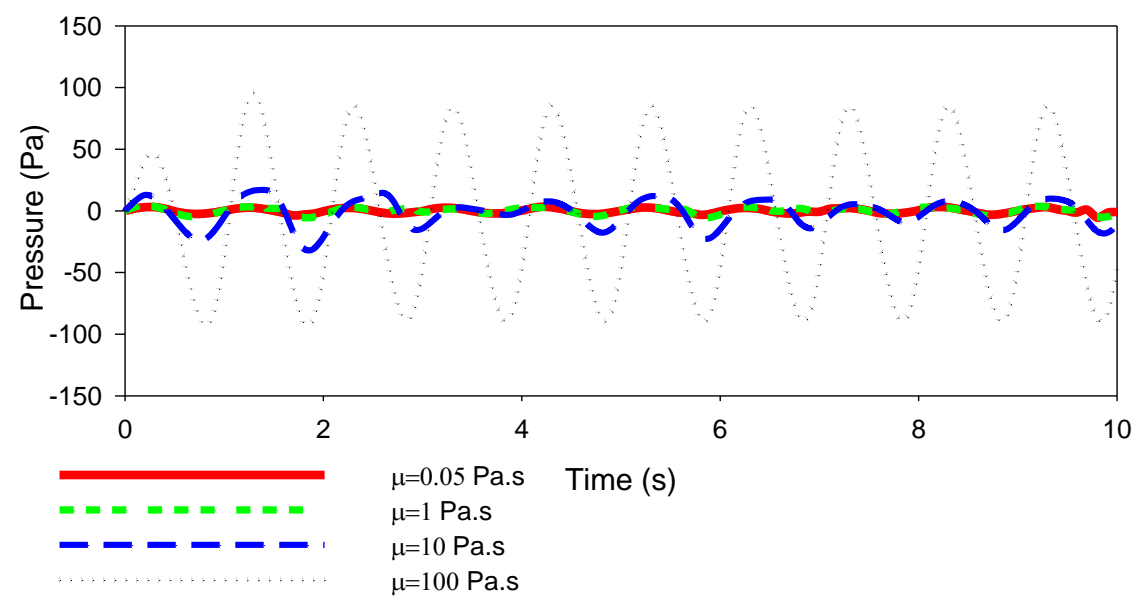

Fig. 12. Changing pressure values for the pitch angle $\theta_{0}=4^{\circ}$ at the different values of viscosity. The case of a sinusoidal density form. 


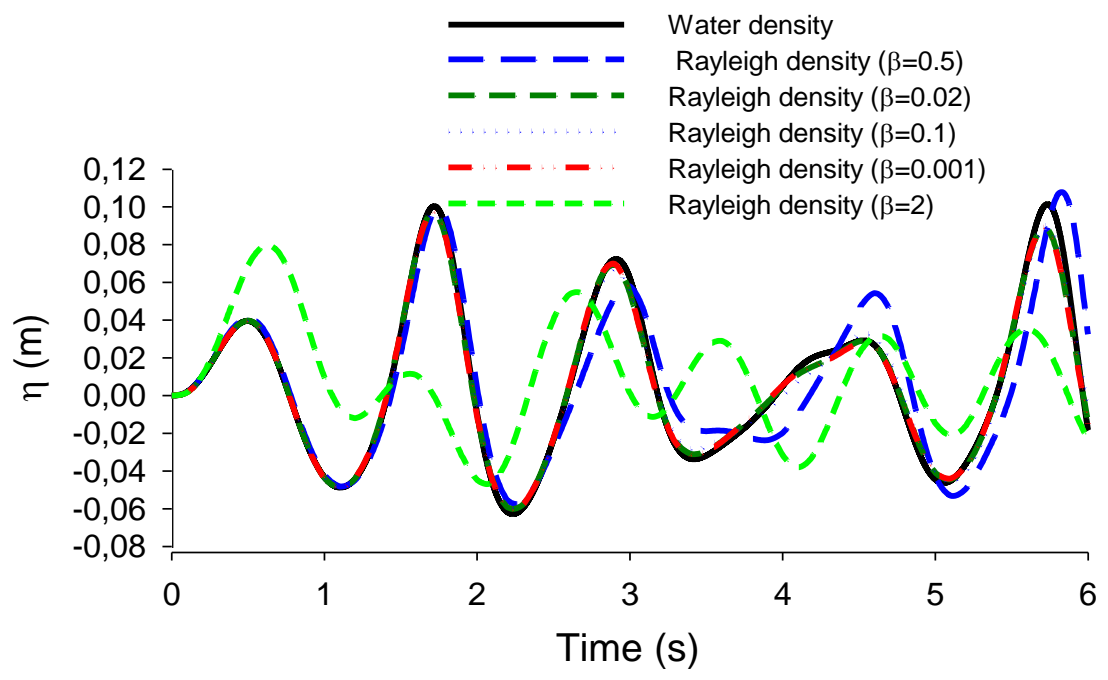

Fig. 13. Time simulation of the free surface profile, effect of the $\beta$ parameter (case of the Rayleigh density).

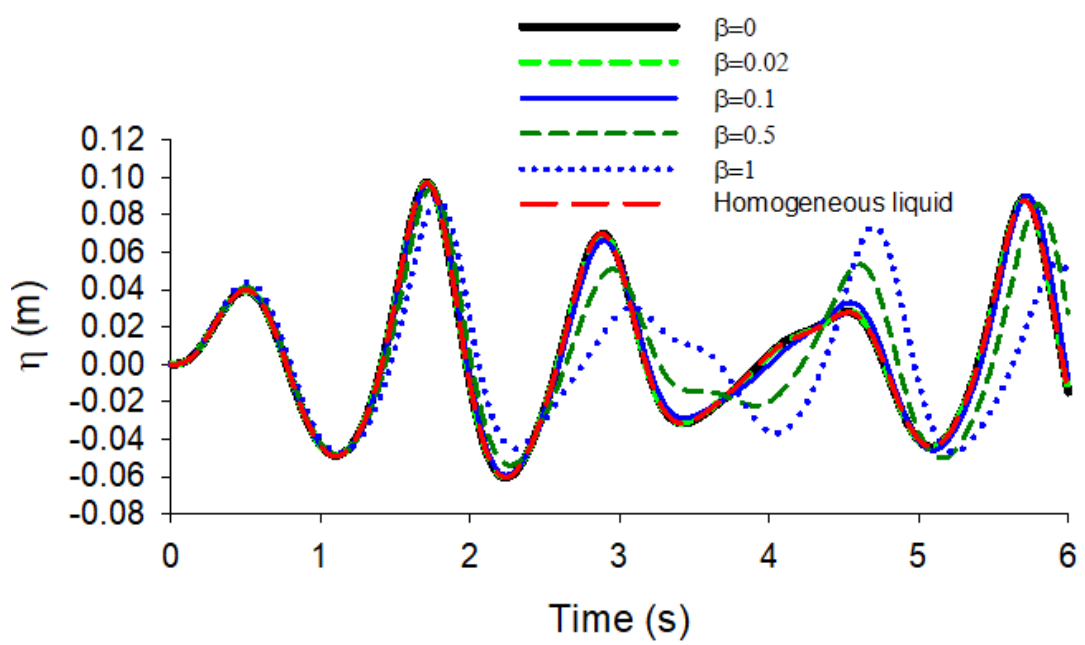

Fig. 14. Time simulation of the free surface profile, effect of the $\beta$ parameter (case of the Almost Homogeneous Liquid).

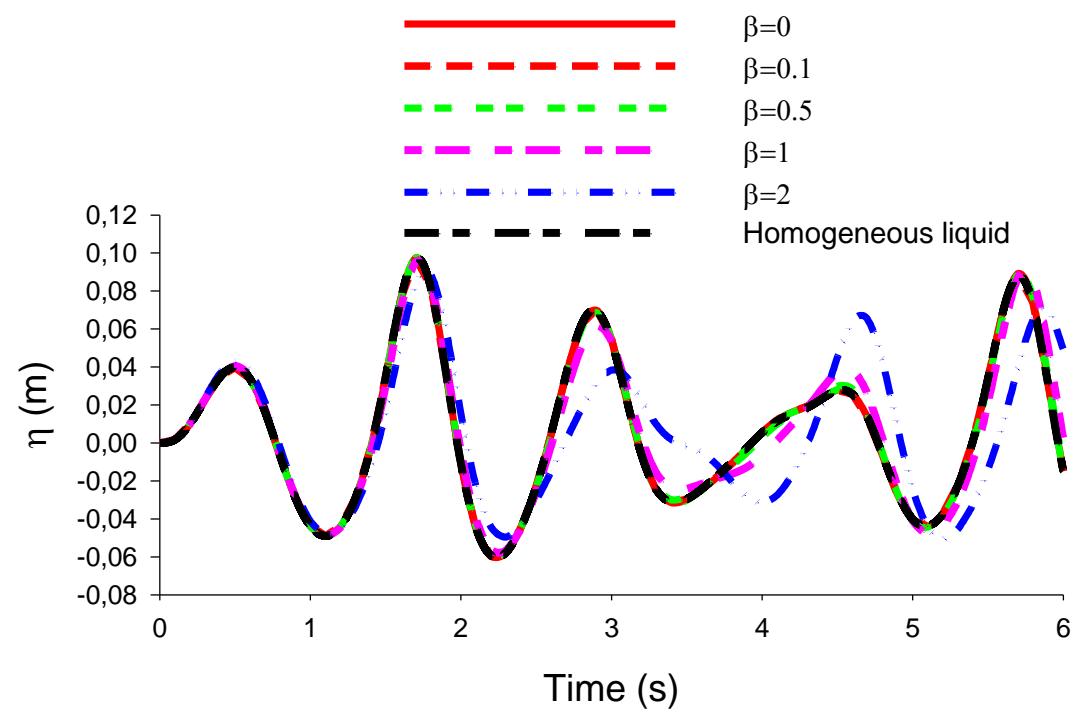

Fig. 15. Time simulation of the free surface profile, effect of the $\beta$ parameter (a sinusoidal density). 
J. El Bahaoui et al. / JAFM, Vol. 13, No. 5, pp. 1391-1405, 2020.

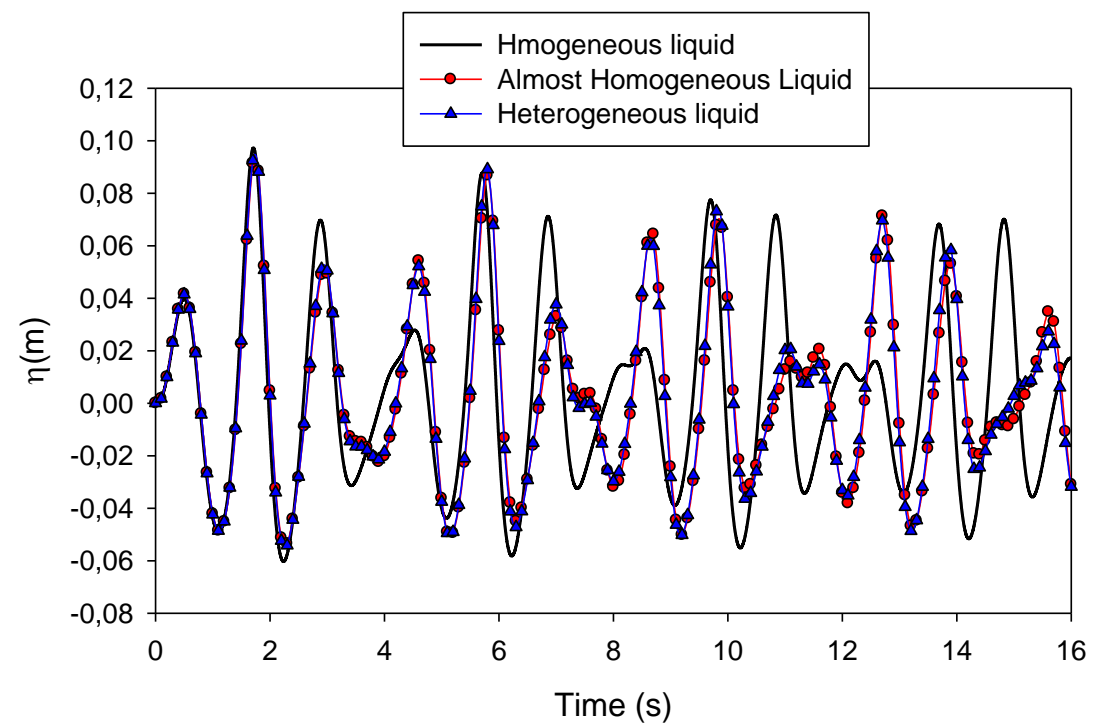

Fig. 16. Comparison of the homogeneous and heterogeneous sloshing analysis with various parameters.

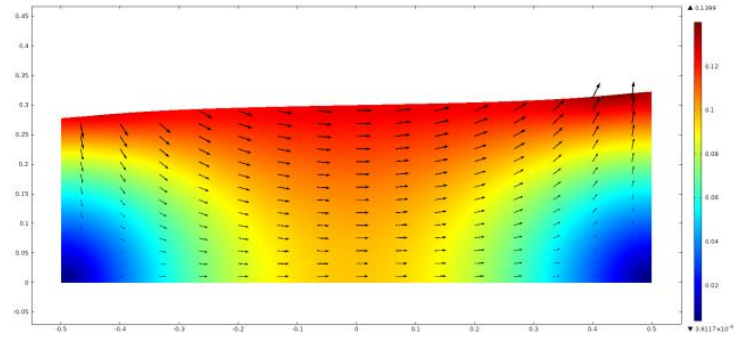

$t=0.3$ s vis $=0.3$ Pa.s homogenious liquid

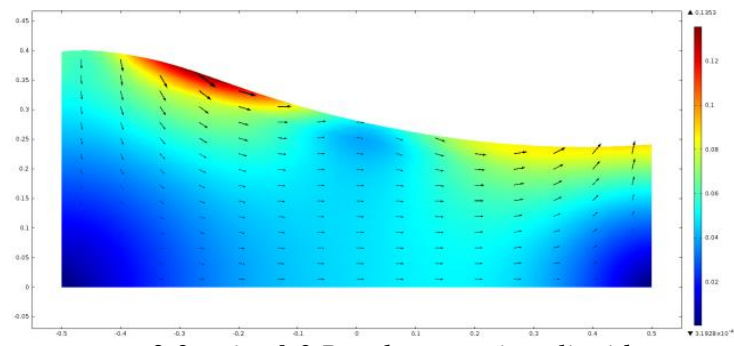

$t=2.3 \mathrm{~s}$ vis $=0.3$ Pa.s homogenious liquid

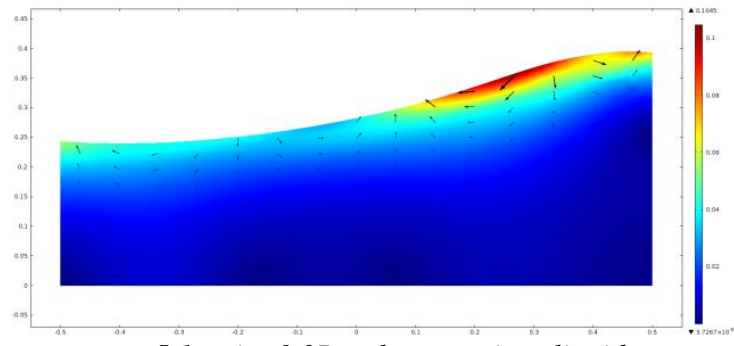

$t=5.1 \mathrm{~s}$ vis=0.3Pa.s homogenious liquid

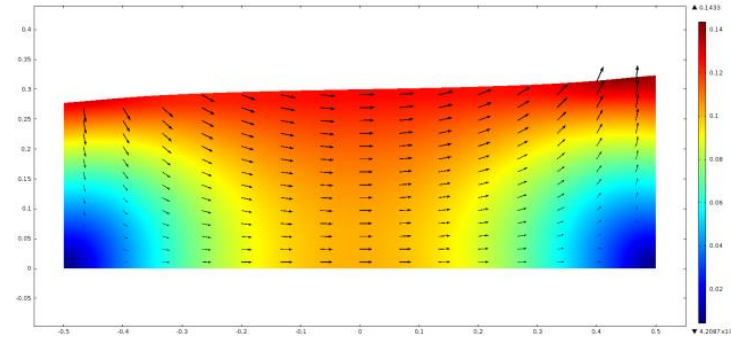

$t=0.3$ s vis $=0.3$ Pa.s heterogenious liquid

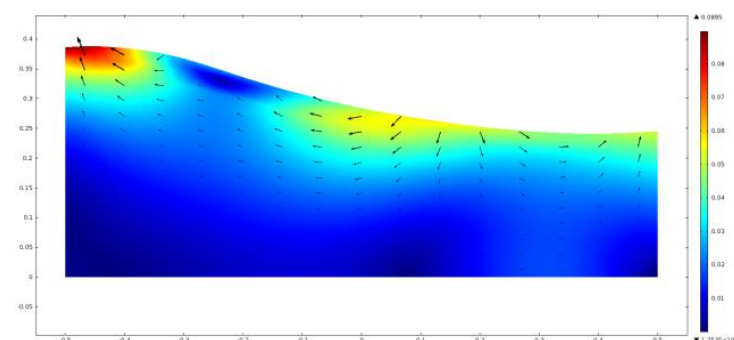

$t=2.3$ s vis $=0.3$ Pa.s heterogenious liquid

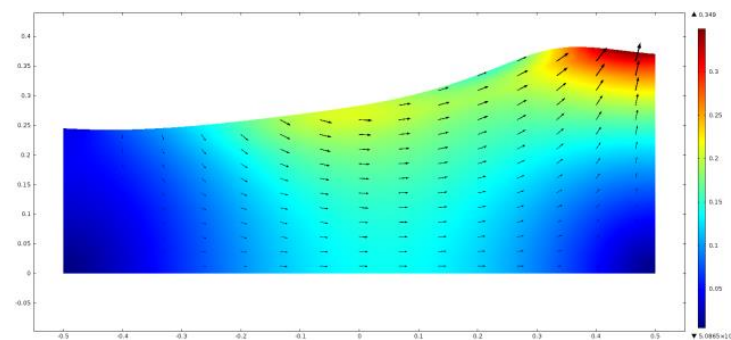

$t=5.1 \mathrm{~s}$ vis $=0.3$ Pa.s heterogenious liquid

Fig. 17. A snapshots for numerical simulation of the shallow liquid sloshing free surface (Velocity field for different time in the homogenous and heterogeneous liquid). 
After the figures, we can see that in the case where the parameter beta tends to zero, we find ourselves in the case of a homogeneous liquid (water density). This result is still valid for the different density forms considered.

Figure 16 show the comparison of the homogeneous and heterogeneous sloshing analysis with various viscosities. Watching the nature of the curves, the behavior of tested liquid is almost the same, this is valid for a time variation of only $3 \mathrm{~s}$. After the behavior profile changes and the important effect of heterogeneity appears.

\subsection{Snapshots of the Velocity and Pressure}

For investigating the nonlinear effects of fluid motion in real scale tanks. the numerical modeling of the fluid motion including heterogeneity effects on the free surface elevation is introduced in this section. The nonlinear numerical model is considered the finite element method is utilized using a 2-D fluid element identified in COMSOLMultiphysics. Figure 17 denote the snapshots of the liquid motion including the free surface deformation, and displays the

numerical results for the time step size $0.3 \mathrm{~s}, 2.3 \mathrm{~s}$ and 5.2s. We are interested on the velocity field, the Fig. 18 denote the snapshots of the liquid motion including the free surface deformation of pressure for different time step size $0.3 \mathrm{~s}, 2.3 \mathrm{~s}$ and $5.2 \mathrm{~s}$ in the homogenous and heterogeneous liquid.

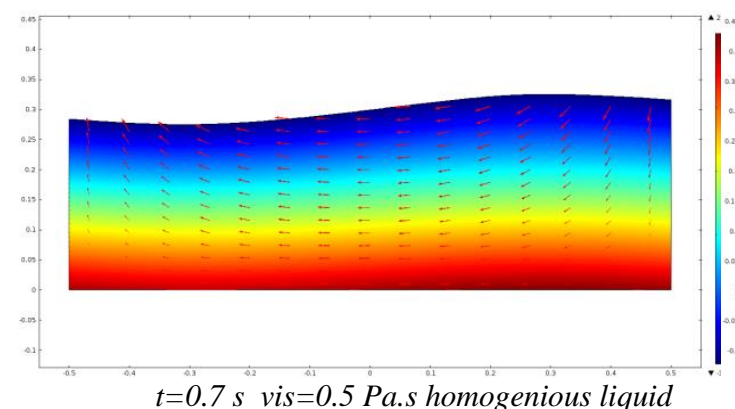

$t=0.7 \mathrm{~s}$ vis $=0.5$ Pa.s homogenious liquid

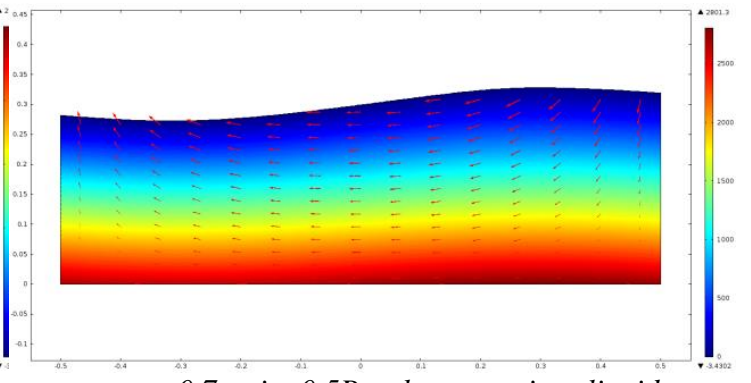

$t=0.7 \mathrm{~s}$ vis $=0.5$ Pa.s heterogenious liquid

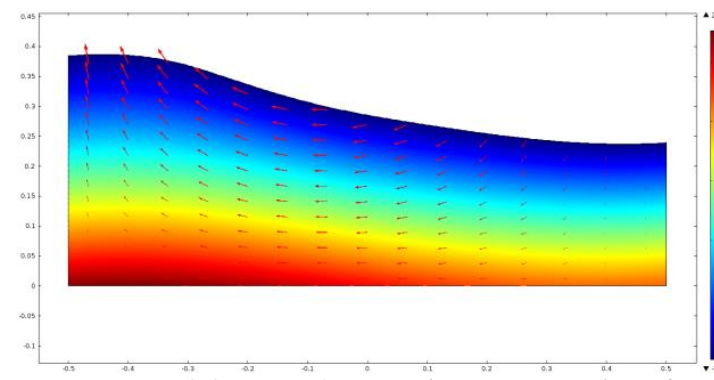

$t=2.2 \mathrm{~s}$ vis=0.5 Pa.s homogenious liquid

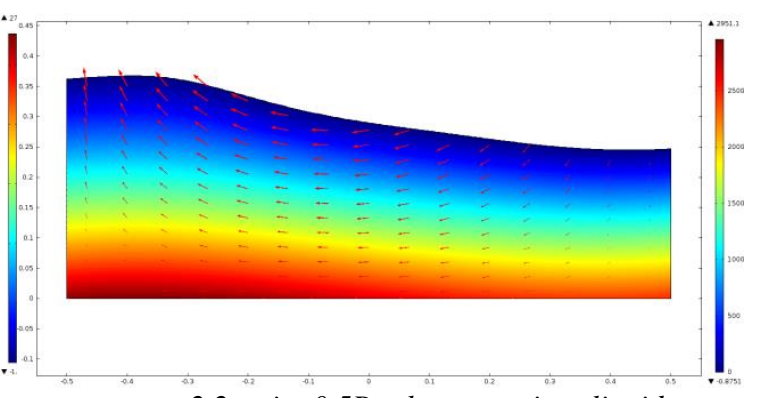

$t=2.2 \mathrm{~s}$ vis $=0.5$ Pa.sheterogenious liquid
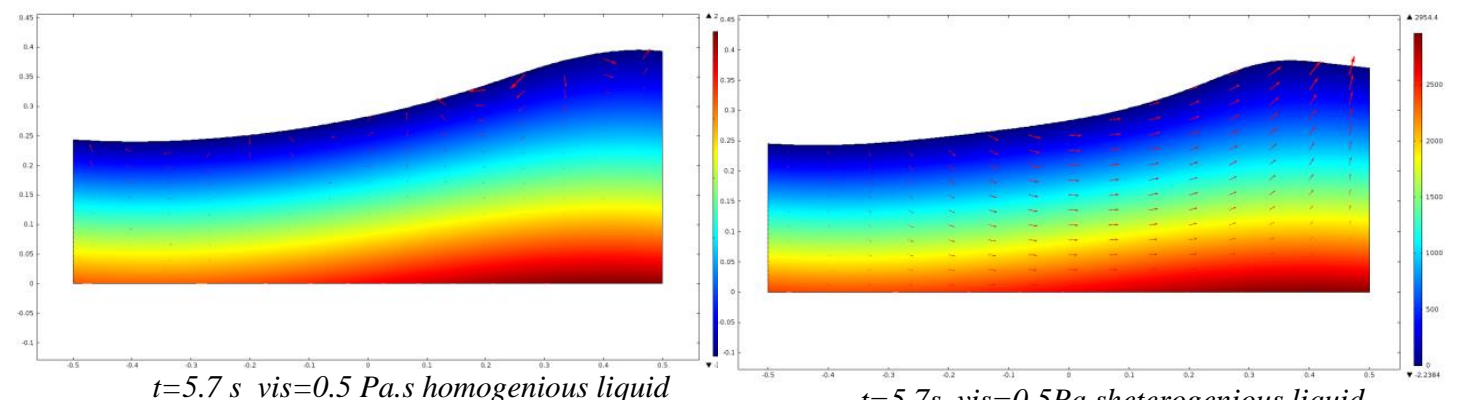

$t=5.7 \mathrm{~s}$ vis $=0.5$ Pa.sheterogenious liquid

Fig. 18. A snapshot for numerical simulation of pressure for different time in deep homogenous and heterogeneous liquid.

According to these results, it can be seen from there that the maximum free surface deformation is obtained at $\mathrm{t}=2.2 \mathrm{~s}$. it has been noted that a liquid behavior and almost the same in two cases homogenous and heterogeneous liquid. The results showed that the motion of the liquid becomes more important time. For the case the heterogeneous liquid, it has been observed that the motion of the liquid is a little weak. For the second case of the considered liquid, the liquid 
sloshing becomes more important and the liquid reached the top of the tank.

\section{CONCLUSION}

In this study, the effects of the variable density liquid are investigated based on the liquid sloshing in a moving partially filled 2-Dractangular tank under pitching excitation. The free Surface deformation is presented at different instances. A numerical model based on the finite element of fluid is used to study the nonlinear behavior of heterogeneity liquid sloshing. This model solves the complete Navier-Stokes equations. The Model was used to compare the effect of viscosity on the pressure exerted by the liquid. It is proved that the presence of viscosity removes the specter and free surface elevation profile which appears in the case of a heterogeneous liquid. The small damped motions of the considered system depend on the viscosity coefficient and variable density of liquid.

The effect of viscosity is predominant on the vibration of a heterogeneous liquid containing in the rectangular tank under pitching excitation. Liquid sloshing is a result of the motion of the partially filled tank. This motion mainly depends on several parameters such as the density of fluid. In the case of resonance when the frequency of the tank motion is close to one of the natural frequencies of the fluid, our study shows that heterogeneity is a very important parameter and may lead to the phenomenon of resonance.

\section{ACKNOWLEDGMENTS}

The research is supported by the M2SM Laboratory ER28/FS/05, Abdelmalek Essaadi University, Tetouan, Morocco.

\section{REFERENCES}

Akyildiz, H. (2012), A numerical study of the effects of the vertical baffle on liquid sloshing in two-dimensional rectangular tank. Journal of Sound and Vibration 331, 41-52

Brar, G. S. and S. Singht (2014). An experimental and CFD analysis of sloshing in tanker, $2^{N D}$ International conference on innovations in automation and mechatronics engineering. ICIAME 2014. Procedia Technology 14, 490496. Elsevier.

Capodanno, P. (1993) Un exemple simple de problème non standard de vibration: oscillations d'un liquide hétérogène pesant dans un container, Mechanics Research Communications 20(3), 257-261,

Capodanno, P. (1996), Etude mathématique des petites oscillations de deux liquides pesants non miscibles dans un container, Mechanics Research Communications 23(1) 75-80.

Capodanno, P. (2001) Piccole oscillazioni piane di un liquido perfetto incompressibile pesante eterogeneo in un recipiente, Lecture in the Rome University TR- CTIT, 10-16.

Chen B. F. and Huang, S. M. (2015). A numerical study of liquid tank and structure interaction; Journal of Marine Science and Technology 23(5), 781-791.

Elahi, R., M. Passandideh-Fard, A. Javanshir (2015) Simulation of liquid sloshing in 2D containers using the volume of fluid method. Ocean Engineering 96, 226-244.

Essaouini, H., J. El Bahaoui, L. El Bakkali and P. Capodanno (2014), Mathematical analysis of the small oscillations of a heavy heterogeneous viscous liquid in an open immovable container. Engineering Mathematics Letters 2014, 2.

Eswaran, M. and K. Ujjwal Saha (2011). Sloshing of liquids in partially filled tanks - a review of experimental investigations. Ocean Systems Engineering 1(2),131-155.

Faltinsen, A. (1978) Numerical Nonlinear Method of Sloshing in Tanks with Two-Dimensional Flow, JSR 22 (3) 193-202.

Faltinsen, O. M. (1974). A nonlinear theory of sloshing in rectangular tanks. Journal of Ship Research 18(4), 224-241.

Godderidge, E., S. Turnock, M. Tan and C. Earl (2009) An investigation of multiphase CFD modelling of a lateral sloshing tank, Computers \& Fluids 38, 183-193

Goudarzi, M. A. and P. Nourae Danesh (2016), Numerical investigation of a vertically baffled rectangular tank under seismic excitation. Journal of Fluids and Structures, 61450-460.

Goudarzi, M. A. and S. R. Sabbagh-Yazdi (2012). Investigation of nonlinear sloshing effects in seismically excited tanks. Soil Dynamics and Earthquake Engineering 43 355-365.

Javanshir, A., R. Elahi and M. Passandideh-Fard (2013) Numerical Simulation of liquid Sloshing with baffles in the fuel container. The 12th Iranian Aerospace Society Conference AERO2013-17469 Amirkabir University of Technology.

Kopachevskii, D. and S. G. Krein (2003) Operator approach to linear problems of Hydrodynamics, Vol 2, Birkhauser, Basel, 2003.

Lamb, H. (1932) Hydrodynamics. Cambridge at the University Press, Cambridge, 1932.

Liu, D. and P. Lin (2008) A numerical study of three-dimensional liquid sloshing in tanks, Journal of Computational 227(8), 3921-3939

Lyu, W., M. El Moctar, R. Potthoff and J. Neugebauer (2017), Experimental and numerical investigation of sloshing using different free surface capturing methods, 
J. El Bahaoui et al. / JAFM, Vol. 13, No. 5, pp. 1391-1405, 2020.

Applied Ocean Research 68, 307-324.

Mirzaii, I. and M. Passandideh-Fard (2012) Modeling free surface flows in presence of an arbitrary moving object. International Journal of Multiphase Flow 39, 216-226.

Nakayama, and K. Washizu (1980) Nonlinear analysis of liquid motion in a container subjected to forced pitching oscillation, International Journal for Numerical Methods in Engineering 15(8) $1207-1220$.

Pal, P. and S. K. Bhattacharyya (2010), Sloshing in partially filled liquid containers Numerical and experimental study for2-D problems. Journal of Sound and Vibration 329, 44664485

Raouf, A. I. (2005); Liquid Sloshing Dynamics: Theory and Applications, Cambridge University Press.

Veldman, A. E. P., J. Gerrits, R. Luppes, J. A.
Helder, J. P. B. Vreeburg (2007) The numerical simulation of liquid sloshing on board spacecraft. Journal of Computational Physics 224 82-99.

Wu, G. X., Q. A. Ma and T. R. Eatock (1998) Numerical simulation of sloshing waves in a 3D tank base, finite element method. Journal of Applied Ocean Research 20, 337-55.

Xue, M. A. and P. Lin (2011). Numerical study of ring baffle effects on reducing violent liquid sloshing. Computers \& Fluids 52, 116-129

Zhang, H. and B. Sun (2014), Numerical simulation of sloshing in 2D rectangular tanks based on the prediction of free surface. Mathematical Problems in Engineering 2014. Hindawi Publishing Corporation.

Zhang, T., Y. F. Ren, C. M. Fan and P. W. Li (2016) Simulation of two dimensional sloshing phenomenon by generalized finite difference method. Engineering Analysis with Boundary Elements. 6382-91. Elsevier. 